\title{
Pathways Involving Beta-3 Adrenergic Receptors Modulate Cold Stress-induced Detrusor Overactivity in Conscious Rats
}

\author{
Tetsuya IMAMURA, ${ }^{1 *}$ Osamu ISHIZUKA, ${ }^{1,2}$ Teruyuki OGAWA, ${ }^{2}$ \\ Takahiro YAMAGISHI, ${ }^{2}$ Hitoshi YOKOYAMA,${ }^{2}$ Tomonori MINAGAWA, ${ }^{2}$ \\ Masaki NAKAZAWA, ${ }^{2}$ and Osamu NISHIZAWA ${ }^{1,2}$ \\ ${ }^{1}$ Department of Lower Urinary Tract Medicine and ${ }^{2}$ Department of Urology, \\ Shinshu University School of Medicine, Matsumoto, Japan
}

\section{${ }^{*}$ Correspondence:}

Tetsuya Imamura, Ph.D.

Department of Lower Urinary Tract Medicine,

Shinshu University School of Medicine,

3-1-1 Asahi, Matsumoto, Nagano, 390-8621, JAPAN

TEL: +81-263-37-2661

FAX: +81-263-37-3082

E-mail: imatetu@shinshu-u.ac.jp

Running Head: Cold stress LUTS and beta-3 ARs

Key words:

beta-3 adrenergic receptor, cold stress, detrusor overactivity, SD rat, lower urinary tract symptoms 


\section{Abstract}

Objective: To investigate pathways involving beta-3 adrenergic receptors (ARs) in detrusor overactivity induced by cold stress, we determined if the beta-3 AR agonist CL316243 could modulate the cold stress-induced detrusor overactivity in normal rats.

Methods: Two days prior to cystometric investigations, the bladders of 10-week-old female Sprague-Dawley rats were cannulated. Cystometric measurements of the unanesthetized, unrestricted rats were taken to estimate baseline values at room temperature $\left(\mathrm{RT}, 27 \pm 2^{\circ} \mathrm{C}\right.$ ) for $20 \mathrm{~min}$. They were then intravenously administered vehicle, $0.1 \mathrm{mg} / \mathrm{kg}$, or $1.0 \mathrm{mg} / \mathrm{kg}$ CL316243 (n=6 in each group). Five minutes after the treatments, they were gently and quickly transferred to the low temperature (LT, $4 \pm 2^{\circ} \mathrm{C}$ ) room for 40 min where the cystometric measurements were again made. Afterward, the rats were returned to RT for final cystometric measurements. The cystometric effects of CL316243 were also measured at RT ( $\mathrm{n}=6$ in each group).

Results: At RT, both low and high dose of CL316243 decreased basal and micturition pressure while the high dose $(1.0 \mathrm{mg} / \mathrm{kg})$ significantly increased voiding interval and bladder capacity. During LT exposure, the high dose of CL316243 partially reduced cold stress-induced detrusor overactivity characterized by increased basal pressure and urinary frequency. The high drug dose also significantly inhibited the decreases of both voiding interval and bladder capacity compared to the vehicle- and low dose $(0.1$ $\mathrm{mg} / \mathrm{kg}$ )-treated rats.

Conclusion: A high dose of the beta-3 agonist CL316243 could modulate cold stress-induced detrusor overactivity. Therefore, one of the mechanisms in cold stress-induced detrusor overactivity includes a pathway involving beta-3 ARs. 


\section{INTRODUCTION}

Empirically it is well established that cold stress produced by sudden whole body cooling at low environmental temperature elicits urinary sensations and frequent urination in healthy people. For patients with lower urinary tract symptoms (LUTS), seasonal or continuous low environmental temperature is one of the risk factors that exacerbate urinary urgency, frequency, and nocturia. ${ }^{1,2}$ To investigate cold stress-related physiological responses and cold stress-exacerbated LUTS, we have established an animal model with detrusor overactivity induced by cold stress. ${ }^{3}$ A typical cold stress-induced detrusor overactivity pattern is characterized by increased basal pressure, urinary frequency with less than 5-min voiding interval, and less than 1-ml micturition volume. In normal rats, the cold stress-induced detrusor overactivity slowly diminishes over time during low temperature (LT) exposure, and completely disappears after returning to room temperature (RT). We showed that in conscious rats the cold stress-induced detrusor overactivity was partially mediated by resiniferatoxin-sensitve (C-fiber) sensory ${ }^{3}$ and sympathetic neurological pathways. ${ }^{4}$

In the urinary bladder, beta-3 adrenergic receptors (ARs) mediate sympathetic relaxation of the urinary detrusor. ${ }^{5-8}$ Thus, beta-3 AR agonists promote urine storage and improve LUTS such as urinary urgency, frequency, and/or incontinence. ${ }^{9}$ We determined if the beta-3 AR agonist CL316243 could inhibit the cold stress-induced detrusor overactivity in normal rats. In addition, we investigated the pathways involving beta-3 ARs in the cold stress-induced responses. 


\section{METHODS}

\subsection{Animals}

Forty-two 10-week-old female Sprague-Dawley rats (Japan SLC Inc., Shizuoka, Japan) were used for the experiments. The animals were treated in accordance with National Institutes of Health Animal Care Guidelines and the guidelines approved by the Animal Ethics Committee of Shinshu University School of Medicine.

\subsection{Drug}

We used 5-[(2R)-2-[[(2R)-2-(3-Chlorophenyl)-2-hydroxyethyl]amino]propyl] -1,3-bezodioxole-2,2-dicarboxylic acid disodium salt (CL316243; molecular weight: 465.8; Sigma, St. Louis, MO, USA) as a beta-3 AR agonist. CL316243 is potent and highly selective for beta3-AR [ $\mathrm{EC}_{50}=3 \mathrm{nM}$ (rat), $\mathrm{Ki}=20 \mu \mathrm{M}$ (human)] compared to beta-1 AR [ $\mathrm{IC}_{50}=30 \mu \mathrm{M}$ (rat), $\mathrm{Ki}=100 \mu \mathrm{M}$ (human)] and beta2-AR [ $\mathrm{IC}_{50}=9.77 \mu \mathrm{M}$ (rat), $\mathrm{Ki}=79.2 \mu \mathrm{M}$ (human)]. The CL316243 was dissolved in $0.9 \%$ saline and diluted to the desired concentration with $0.9 \%$ saline.

\subsection{Cystometric investigations}

The animals were anesthetized with pentobarbital sodium solution (40 mg/kg-body weight, Kyoritsu Seiyaku Co., Tokyo, Japan), and the urinary bladders were exposed and incised at the center of the dome. A polyethylene catheter (PE50, Becton Dickinson and Company, Sparks, MD, USA) was inserted through the incision and fixed at that site with a 5-0 suture. The free end was tunneled subcutaneously and exteriorized at the back of the neck. The cannulated rats were kept for 2 days as above.

Two days after the cannulation, a polyethylene catheter (PE10, Becton Dickinson and Company) was inserted into the jugular vein of rats anesthetized by inhalation of 3\% sevoflurane (Abbot Japan Co., Ltd., Tokyo, Japan). Each rat was allowed to recover from the anesthesia in a metabolic cage for 2 hours. After the recovery, the bladder 
catheter was connected through a T-tube to a pressure transducer (P23 DC; Nihon Kohden, Tokyo, Japan) and a syringe pump (TE-351, Terumo, Tokyo, Japan). To measure micturition volume, a fluid collector connected to a force displacement transducer (Type 45196; NEC San-ei Instruments, Tokyo, Japan) was placed under the metabolic cage. Throughout the experiments, saline kept at room temperature was pumped into the bladder at a rate of $10 \mathrm{ml} / \mathrm{hr}$. The bladder pressure and micturition volume were recorded continuously on a pen oscillograph $(10 \mathrm{~mm} / \mathrm{min}$ recording speed; Recti-Horiz-8K; NEC San-ei Instruments). The following cystometric parameters were measured: basal pressure $\left(\mathrm{cmH}_{2} \mathrm{O}\right)$, micturition pressure $\left(\mathrm{cmH}_{2} \mathrm{O}\right)$, voiding interval (min), micturition volume (ml), residual volume (ml), and bladder capacity (ml). Bladder capacity was calculated by adding the micturition volume and residual volume that was determined as the difference between the saline infusion volume and micturition volume. The rats were not given food and water during the cystometric investigations. After the cystometric investigations, the rats were euthanized by inhalation of diethyl ether.

\subsection{Cystometric investigations without cold stress}

Cystometric investigations of the catheterized, unanesthetized, unrestricted rats were were taken for $20 \mathrm{~min}$ at $\mathrm{RT}, 27 \pm 2^{\circ} \mathrm{C}$ (mean \pm standard error of the mean). They were then intravenously administered with $0.1 \mathrm{mg} / \mathrm{kg}$ or $1.0 \mathrm{mg} / \mathrm{kg}$ CL316243 in $0.2 \mathrm{ml}$ solution volume ( $n=6$ in each group). Five minutes after the treatments, the cystometric measurements were continued for $20 \mathrm{~min}$ at RT.

\subsection{Cystometric investigations with cold stress}

Cystometric measurements of the catheterized, unanesthetized, unrestricted rats were taken under the following environmental temperature conditions. The rats were placed singly in metabolic cages at RT for 20 min during which the first cystometric measurements were made to estimate baseline values. CL316243 $(0.1 \mathrm{mg} / \mathrm{kg}$ or 1.0 $\mathrm{mg} / \mathrm{kg}$ ) or vehicle was then intravenously administered in $0.2 \mathrm{ml}$ solution volume ( $\mathrm{n}=6$ in each group). Five minutes after the treatments, they were gently and quickly transferred to the metabolic cages in the cold room for LT exposure $\left(4 \pm 2^{\circ} \mathrm{C}\right)$ for $40 \mathrm{~min}$ 
during which the cystometry continued. For analysis, the LT exposure was divided into Phase I and Phase II, each of which was $20 \mathrm{~min}$. Afterward, the rats were gently and quickly returned to a RT room for the final 20 min of cystometry.

\subsection{Statistical analysis}

The results were expressed as means \pm standard error of the means. Two-way repeated measures analysis of variance (ANOVA) followed by the Scheffe's test were used within each group. To compare $0.1 \mathrm{mg} / \mathrm{kg}, 1.0 \mathrm{mg} / \mathrm{kg}$ CL316243- and vehicle-treated rats, two-way non-repeated ANOVA followed by the Scheffe's test was used. Differences with $\mathrm{P}<0.05$ were considered significant. 


\section{RESULTS}

\subsection{Effects of beta-3 agonist on micturition parameters without cold stress}

For rats maintained at RT, treatments with CL316243 significantly decreased both basal and micturition pressures (Table 1), while micturition volume and residual volume did not change. The low dose of CL316243, $0.1 \mathrm{mg} / \mathrm{kg}$, did not increase voiding interval and bladder capacity; however, the high dose, $1.0 \mathrm{mg} / \mathrm{kg}$, significantly increased both parameters compared to the pre-treatment values and compared to the low dose-treatment values.

\subsection{Effects of beta-3 agonist on cold stress-induced detrusor overactivity}

During the first 20 min after transfer from RT to LT, Phase I, the vehicle-treated rats exhibited typical cold stress-induced detrusor overactivity patterns (Fig. 1A). However, treatment with the high dose of CL316243 partially reduced the cold stress-induced detrusor overactivity patterns (Fig. 1B).

During Phase I of LT exposure, basal pressure in the vehicle-treated rats increased significantly (Table 2). For rats treated with either low or high dose of CL316243, the basal pressure increased slightly, but the changes were not significant. Thus both doses effectively reduced the increase in basal pressure brought on by exposure to LT during Phase I. After the return to RT (second RT), the basal pressure in each treated rat significantly decreased compared to the LT exposure, and recovered to the baseline level of the first RT. The micturition pressure in vehicle-treated rats did not change throughout the cystometric investigations (Table 2). During the first RT measurements, while the micturition pressure of low dose-treated rats was not different from the vehicle-treated rats, the micturition pressure of high dose-treated rats was significantly lower than that of the vehicle-treated ones (Table 2). The micturition pressures in either low or high dose of CL316243-treated rats during both phases of LT and upon return to RT were lower than the vehicle-treated rats, but the differences were not significant. Also, the micturition pressures in either low or high dose of CL316243-treated rats did not change throughout the cystometric investigations (Table 2). 
Voiding interval and bladder capacity in the vehicle-treated rats decreased significantly during Phase I, and increased significantly during Phase II (Fig. 2A and B). For rats treated with either low or high dose of CL316243, these values also decreased significantly compared to first RT (Fig. 2A and B). However, in contrast to the vehicle-treated rats, these values did not change during Phase II (Fig. 2A and B). After the return to RT, both the voiding interval and bladder capacity in all groups increased significantly and recovered to the baseline levels of the first RT.

During LT Phase I, the decreases of voiding interval and bladder capacity in low dose-treated rats tended to be inhibited compared to vehicle-treated rats, but the differences were not statistically significant. In contrast, these decreases in high dose-treated rats were significantly inhibited compared to the vehicle- and low dose-treated rats $(\mathrm{P}<0.01, \mathrm{P}<0.05$, respectively, Figure $2 \mathrm{~A}$ and $\mathrm{B})$. 


\section{DISCUSSION}

In cystometric investigations without LT exposure, the beta-3 agonist CL316243 significantly decreased basal and micturition pressure; however, micturition and residual volume were not affected. The high dose of CL316243, $1.0 \mathrm{mg} / \mathrm{kg}$, significantly increased voiding interval and bladder capacity, while the low dose, $0.1 \mathrm{mg} / \mathrm{kg}$, did not change these values. It has also been reported that CL316243 decreases bladder pressure without affecting urethral pressure. ${ }^{10}$ These results suggest that CL316243 can affect different aspects of bladder functions.

In cystometric investigations with LT exposure, just after transfer to LT, the vehicle-treated rats exhibited typical cold stress-induced detrusor overactivity patterns, including increased basal pressure and decreased voiding interval and bladder capacity. In contrast, the high dose of CL316243 partially inhibited the cold stress-induced detrusor overactivity patterns that occurred just after transfer to LT. During LT exposure, the basal pressure of CL316243-treated rats tended to increase, but the changes were not significant. Compared to the initial RT values, voiding interval and bladder capacity of rats treated with both doses of CL316243 decreased significantly during Phase I. However, the decreased voiding interval and bladder capacity in the high dose CL316243-treated rats were significantly inhibited compared to the vehicle- and low dose-treated rats.

We reported that the cold stress-induced detrusor overactivity in normal rats slowly diminishes over time during LT Phase II. ${ }^{3,11}$ However in the current study, the CL316243-treated rats did not have the micturition patterns induced by cold stress during Phase I or the gradual return towards baseline RT values during Phase II. This pattern was very similar to results in resiniferatoxin-treated rats. ${ }^{3}$ While the mechanism of the beta-3 AR agonist treatments ${ }^{12-14}$ are different from the resiniferatoxin treatments, ${ }^{15-17}$ a common feature of the two is the increase of urine storage. Thus, the partial reduction in the cold stress-induced detrusor overactivity might be caused by the relaxation of the urinary detrusor promoted by CL316243.

We performed cystometric investigation with two doses of CL316243, $0.1 \mathrm{mg} / \mathrm{kg}$ and $1.0 \mathrm{mg} / \mathrm{kg}$. After transfer to LT, the high dose of CL316243 significantly inhibited the decrease of voiding interval and bladder capacity compared to the low dose. This result suggests that the mechanism by which CL316243 affects cold stress-induced 
detrusor overactivity might be mediated by the improvement of urine storage functions such as increased voiding interval and bladder capacity.

Our cold stress model imitates micturition patterns that simulate human physiological responses due to sudden temperature drops. However, our model has limitations in the investigation of effects associated with CL316243. It is difficult to identify the target areas or target tissues for CL316243 due to the systemic nature of whole body cooling. In this study, we showed that CL316243 affected RT bladder functions by decreasing basal and micturition pressures and increasing voiding interval and bladder capacity. Also, similar urinary bladder effects were reported in rat disease models such as cerebral infarct ${ }^{12}$ and spontaneous hypertension. ${ }^{14}$ These results suggest that CL316243 might affect certain aspects of bladder functions. In contrast, there is a report that CL316243 decreases c-fos expression within the L6 spinal cord, ${ }^{13}$ suggesting that CL316243 could affect the central nervous system as well as the urinary bladder. Thus, while we could not identify the specific target areas of CL316243, it clearly affected mechanisms involving the micturition reflex pathways.

In conclusions, the beta-3 agonist CL316243 partially reduced cold stress-induced detrusor overactivity. Therefore, pathways involving beta-3 ARs could modulate the responses induced by cold stress. If the current results hold true in humans, selective beta-3 AR agonists may provide useful treatments for cold stress-exacerbated LUTS. 


\section{Disclosure}

The authors of the present paper have no financial or commercial interests to disclose. 


\section{REFERENCES}

1. Watanabe T, Maruyama S, Maruyama Y et al. Seasonal changes in symptom score and uroflowmetry in patients with lower urinary tract symptoms. Scand J Urol Nephrol 2007; 41: 521-6.

2. Inoue $\mathrm{H}$, Ishizuka $\mathrm{O}$, Imamura $\mathrm{T}$ et al. Relationship between toe temperature and lower urinary tract symptoms. LUTS 2012; 4: 144-9.

3. Imamura $\mathrm{T}$, Ishizuka $\mathrm{O}$, Aizawa $\mathrm{N}$ et al. Cold environmental stress induces detrusor overactivity via resiniferatoxin-sensitive nerves in conscious rats. Neurourol Urodyn 2008; 27: 348-52.

4. Chen Z, Ishizuka O, Imamura $\mathrm{T}$ et al. Role of alpha1-adrenergic receptors in detrusor overactivity induced by cold stress in conscious rats. Neurourol Urodyn 2009; 28: 251-6.

5. Igawa $\mathrm{Y}$, Yamazaki $\mathrm{Y}$, Takeda $\mathrm{H}$ et al. Relaxant effects of isoproterenol and selective beta3-adrenoceptor agonists on normal, low compliant and hyperreflexic human bladders. J Urol 2001; 165: 240-4.

6. Michel MC, Barendrecht MM. Physiological and pathological regulation of the autonomic control of urinary bladder contractility. Pharmacol Ther 2008; 117: 297-312.

7. Takeda H, Yamazaki Y, Akahane M, Igawa Y, Ajisawa Y, Nishizawa O. Role of the beta(3)-adrenoceptor in urine storage in the rat: comparison between the selective beta(3)-adrenoceptor agonist, CL316, 243, and various smooth muscle relaxants. J Pharmacol Exp Ther 2000; 293: 939-45.

8. Takeda M, Obara K, Mizusawa T et al. Evidence for beta3-adrenoceptor subtypes in relaxation of the human urinary bladder detrusor: analysis by molecular biological and pharmacological methods. J Pharmacol Exp Ther 1999; 288: 
1367-73.

9. Andersson KE, Martin N, Nitti V. Selective beta(3)-adrenoceptor agonists for the treatment of overactive bladder. J Urol 2013; 190: 1173-80.

10. Takeda H, Matsuzawa A, Igawa $\mathrm{Y}$ et al. Functional characterization of beta-adrenoceptor subtypes in the canine and rat lower urinary tract. J Urol 2003; 170: 654-8.

11. Imamura $\mathrm{T}$, Ishizuka $\mathrm{O}$, Sudha $\mathrm{GS}$ et al. A galenical produced from Ba-Wei-Die-Huang-Wan (THC-002) provides resistance to the cold stress-induced detrusor overactivity in conscious rats. Neurourol Urodyn 2013; 32: 486-92.

12. Kaidoh K, Igawa $\mathrm{Y}$, Takeda $\mathrm{H}$ et al. Effects of selective beta2 and beta3-adrenoceptor agonists on detrusor hyperreflexia in conscious cerebral infarcted rats. J Urol 2002; 168: 1247-52.

13. Nagabukuro $\mathrm{H}$, Degenhardt A, Villa $\mathrm{KL}$ et al. Correlation between pharmacologically-induced changes in cystometric parameters and spinal c-Fos expression in rats. Auton Neurosci 2010; 156: 19-26.

14. Patra PB, Thorneloe KS. Enhanced sensitivity to afferent stimulation and impact of overactive bladder therapies in the conscious, spontaneously hypertensive rat. $\mathrm{J}$ Pharmacol Exp Ther 2011; 338: 392-9.

15. Cruz CD, Avelino A, McMahon SB, Cruz F. Increased spinal cord phosphorylation of extracellular signal-regulated kinases mediates micturition overactivity in rats with chronic bladder inflammation. Eur J Neurosci 2005; 21: 773-81.

16. Cruz CD, Charrua A, Vieira E, Valente J, Avelino A, Cruz F. Intrathecal delivery of resiniferatoxin (RTX) reduces detrusor overactivity and spinal expression of TRPV1 in spinal cord injured animals. Exp Neurol 2008; 214: 301-8. 
17. Yokoyama $\mathrm{O}$, Yusup $\mathrm{A}$, Oyama $\mathrm{N}$ et al. Improvement of bladder storage function by alpha1-blocker depends on the suppression of C-fiber afferent activity in rats. Neurourol Urodyn 2006; 25: 461-7. 


\section{Figure Legends}

Figure 1. Typical changes of micturition patterns induced by exposure to low temperature. (A) After transferring from RT to LT Phase I, the vehicle-treated rats exhibited detrusor overactivity patterns that increased urinary frequency. (B) During Phase I, treatment by $1.0 \mathrm{mg} / \mathrm{kg}$ CL316243 partially reduced the cold stress-induced detrusor overactivity patterns. Arrows, voiding points during the first 20 min (Phase I) LT exposure.

Figure 2. Changes of voiding interval and bladder capacity in each cystometric period. In the vehicle-treated rats, both voiding interval and bladder capacity significantly decreased during Phase I, and then significantly increased during Phase II. The voiding interval and bladder capacity at both doses of CL316243 also significantly decreased in LT Phase I compared to RT; however they did not increase significantly in Phase II as occurred in the vehicle-treated rats. After return to RT, the voiding interval and bladder capacity in all groups recovered to the baseline RT period. During Phase I, decreases of both voiding interval and bladder capacity in the high dose-treated rats were significantly inhibited compared to the vehicle- and low dose-treated rats. ${ }^{*} \mathrm{P}<0.01$ compared to first RT in each group; $\uparrow \mathrm{P}<0.01$ compared to Phase I in each group; $\S \mathrm{P}<0.01$ compared to Phase II in each group; $\# \mathrm{P}<0.05$ compared to vehicle- and low dose-treated rats. 
A

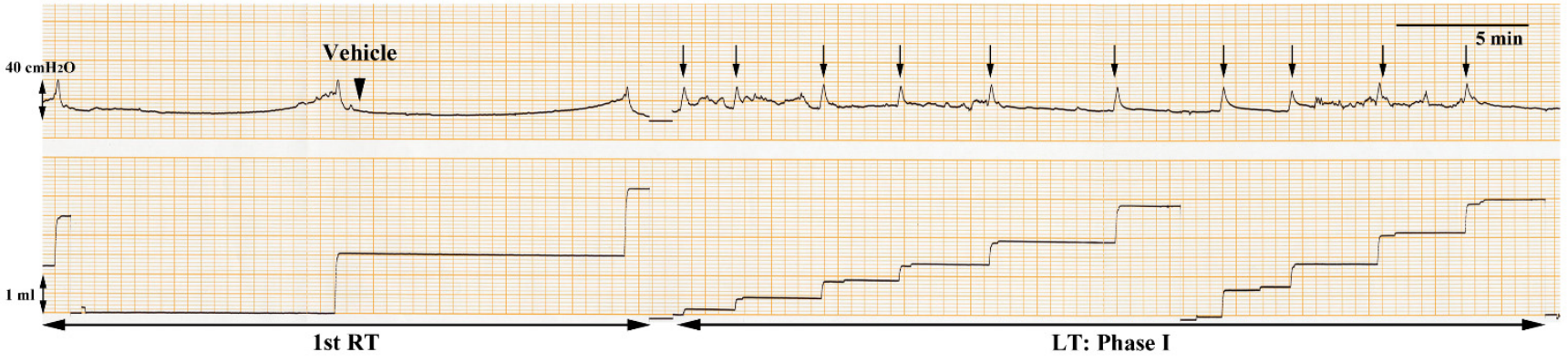

B
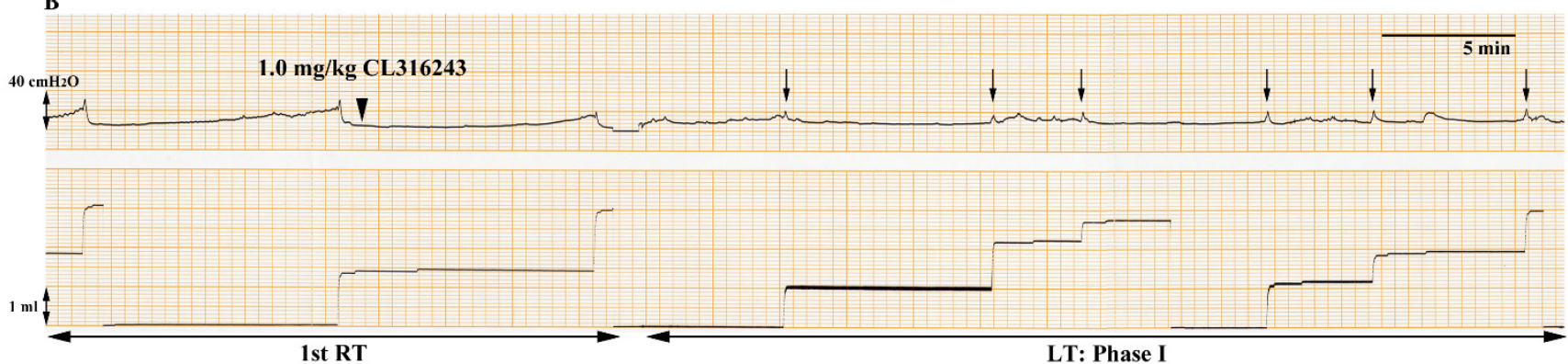

Fig. 1 

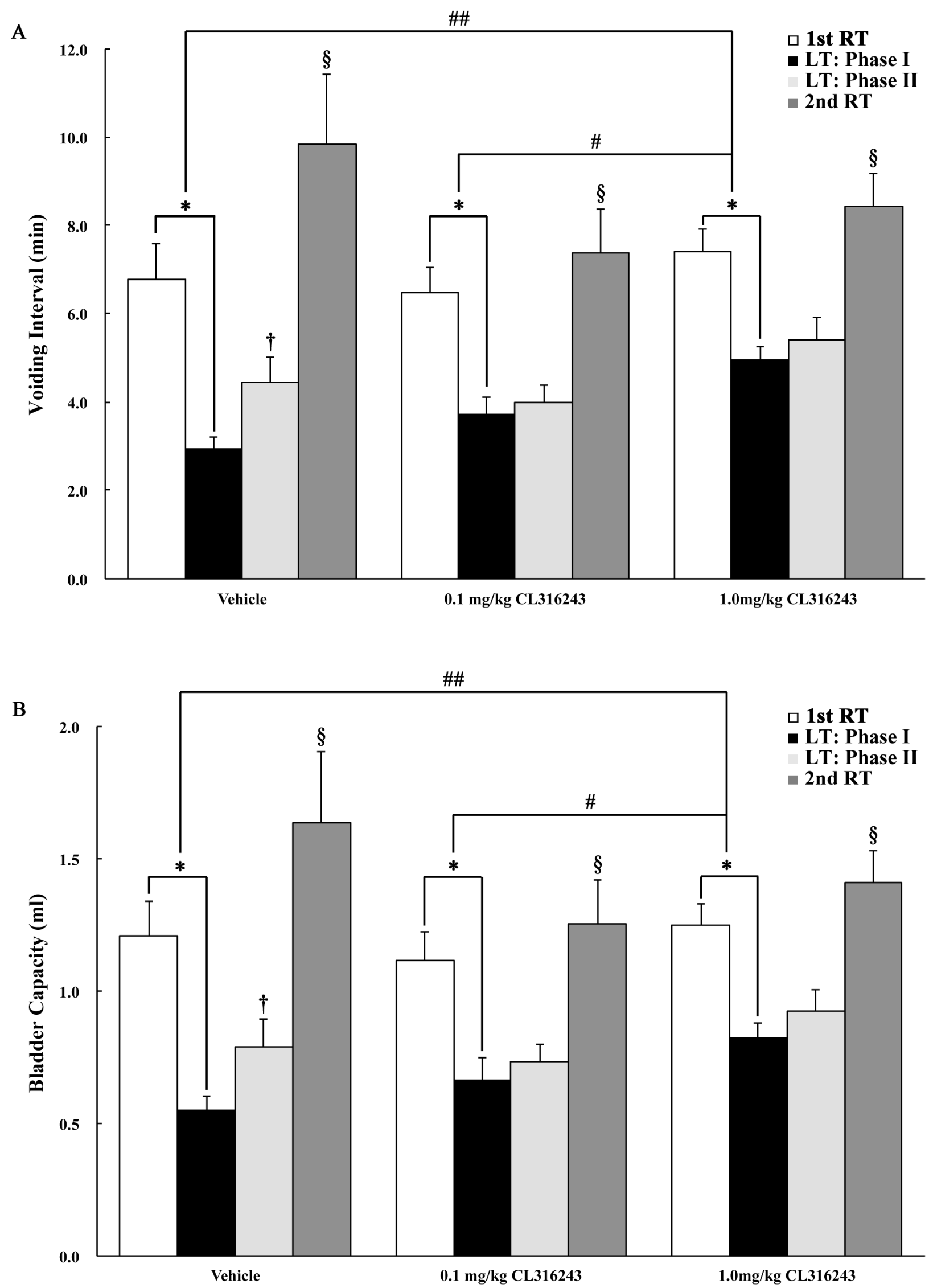

Fig. 2 
TABLE 1. Effects of beta-3 agonist CL316243 on micturition parameters at room temperature

\begin{tabular}{lcccc}
\hline & \multicolumn{2}{c}{$0.1 \mathrm{mg} / \mathrm{kg}$ CL316243 } & \multicolumn{2}{c}{$1.0 \mathrm{mg} / \mathrm{kg}$ CL316243 } \\
\cline { 2 - 5 } & Pre-treatment & Post-treatment & Pre-treatment & Post-treatment \\
\hline Basal pressure $\left(\mathrm{cmH}_{2} \mathrm{O}\right)$ & $8.72 \pm 1.65$ & $6.64 \pm 1.56^{*}$ & $8.51 \pm 1.65$ & $6.63 \pm 1.51^{*}$ \\
Micturition pressure $\left(\mathrm{cmH} \mathrm{H}_{2} \mathrm{O}\right)$ & $40.00 \pm 5.00$ & $24.77 \pm 6.22^{*}$ & $33.13 \pm 2.04$ & $25.24 \pm 2.07 * *$ \\
Voiding interval (min) & $6.59 \pm 0.70$ & $6.33 \pm 0.48$ & $6.98 \pm 0.41$ & $8.13 \pm 0.40^{* * \dagger}$ \\
Micturition volume (ml) & $1.08 \pm 0.13$ & $1.02 \pm 0.12$ & $1.09 \pm 0.05$ & $1.18 \pm 0.07$ \\
Residual volume (ml) & $0.05 \pm 0.01$ & $0.08 \pm 0.03$ & $0.11 \pm 0.04$ & $0.17 \pm 0.03$ \\
Bladder capacity (ml) & $1.13 \pm 0.12$ & $1.10 \pm 0.11$ & $1.20 \pm 0.06$ & $1.36 \pm 0.07 * \dagger$ \\
\hline
\end{tabular}

*P $<0.05,{ }^{* *} \mathrm{P}<0.01$; compared to pre-treatment values

$\dagger \mathrm{P}<0.05$; compared to $0.1 \mathrm{mg} / \mathrm{kg}$ CL316243-treated rats

TABLE 2. Effects of beta-3 agonist CL316243 on bladder pressures with cold stress

\begin{tabular}{lcccccc}
\hline & \multicolumn{3}{c}{ Basal pressure $\left(\mathrm{cmH}_{2} \mathrm{O}\right)$} & \multicolumn{2}{c}{ Micturition pressure (cmH2O) } \\
\cline { 2 - 7 } & Vehicle & $0.1 \mathrm{mg} / \mathrm{kg} \mathrm{CL316243}$ & $1.0 \mathrm{mg} / \mathrm{kg} \mathrm{CL316243}$ & Vehicle & $0.1 \mathrm{mg} / \mathrm{kg}$ CL316244 & $1.0 \mathrm{mg} / \mathrm{kg} \mathrm{CL316244}$ \\
\hline 1st RT & $10.20 \pm 1.27$ & $7.68 \pm 1.59$ & $8.78 \pm 1.76$ & $40.61 \pm 2.65$ & $34.96 \pm 3.06$ & $29.20 \pm 1.69 \S$ \\
LT: Phase I & $15.80 \pm 1.08^{*}$ & $9.97 \pm 1.90$ & $13.62 \pm 2.50$ & $44.35 \pm 3.62$ & $35.25 \pm 4.06$ & $32.22 \pm 4.51$ \\
LT: Phase II & $17.44 \pm 1.59$ & $11.51 \pm 2.31$ & $12.32 \pm 1.87$ & $47.76 \pm 3.70$ & $41.55 \pm 4.06$ & $35.83 \pm 6.32$ \\
2nd RT & $11.64 \pm 2.48 \ddagger$ & $5.39 \pm 1.41 \ddagger$ & $7.48 \pm 2.18 \dagger$ & $39.10 \pm 6.91$ & $33.49 \pm 5.5 .92$ & $26.26 \pm 2.21$ \\
\hline
\end{tabular}

$* \mathrm{P}<0.01$ compared to initial $\mathrm{RT} ; \dagger \mathrm{P}<0.05, \ddagger \mathrm{P}<0.01$ compared to LT Phase II.

$\S \mathrm{P}<0.05$ compared to vehicle-treated rats; $\mathrm{RT}$, room temperature; $\mathrm{LT}$, low temperature. 